\title{
GRADIENTS OF ODD THETA FUNCTIONS
}

\author{
SAMUEL GRUSHEVSKY AND RICCARDO SALVATI MANNI
}

\begin{abstract}
We show that a generic principally polarized abelian variety is uniquely determined by its gradient theta-hyperplanes, the non-projectivized version of those studied in [Ca01, [CS00, CS02, which in a sense are a generalization to ppavs of bitangents of plane curves. More precisely, we show that, generically, the set of gradients of all odd theta functions at the point zero uniquely determines a ppav with level $(4,8)$ structure. We also show that our map is an immersion of the moduli space of ppavs.
\end{abstract}

\section{Definitions and notations}

We denote by $\mathcal{H}_{g}$ the Siegel upper half-space - the space of complex symmetric $g \times g$ matrices with positive definite imaginary part. An element $\tau \in \mathcal{H}_{g}$ is called a period matrix, and defines the complex abelian variety $X_{\tau}:=\mathbb{C}^{g} / \mathbb{Z}^{g}+\tau \mathbb{Z}^{g}$. The group $\Gamma_{g}:=\operatorname{Sp}(2 g, \mathbb{Z})$ acts on $\mathcal{H}_{g}$ by automorphisms: for $\gamma:=\left(\begin{array}{ll}a & b \\ c & d\end{array}\right) \in \operatorname{Sp}(2 g, \mathbb{Z})$ the action is $\gamma \tau:=(a \tau+b)(c \tau+d)^{-1}$. The quotient of $\mathcal{H}_{g}$ by the action of the symplectic group is the moduli space of principally polarized abelian varieties (ppavs): $\mathcal{A}_{g}:=\mathcal{H}_{g} / \mathrm{Sp}(2 g, \mathbb{Z})$. A ppav is called irreducible if it is not a direct product of two lower-dimensional ppavs, i.e. if its period matrix $\tau$ is not conjugate by the action of $\Gamma_{g}$ to a matrix that splits as $\tau_{1} \oplus \tau_{2}$ for two lower-dimensional period matrices. For us the case $g=1$ is special and in the following we will always assume $g>1$.

We define the level subgroups of the symplectic group to be

$$
\begin{gathered}
\Gamma_{g}(n):=\left\{\gamma=\left(\begin{array}{ll}
a & b \\
c & d
\end{array}\right) \in \Gamma_{g} \mid \gamma \equiv\left(\begin{array}{ll}
1 & 0 \\
0 & 1
\end{array}\right) \bmod n\right\} \\
\Gamma_{g}(n, 2 n):=\left\{\gamma \in \Gamma_{g}(n) \mid \operatorname{diag}\left(a^{t} b\right) \equiv \operatorname{diag}\left(c^{t} d\right) \equiv 0 \bmod 2 n\right\} .
\end{gathered}
$$

The corresponding level moduli spaces of ppavs are denoted $\mathcal{A}_{g}^{n}$ and $\mathcal{A}_{g}^{n, 2 n}$, respectively.

Date: May 11, 2003.

1991 Mathematics Subject Classification. 14K25, 11F23, 14H42, 11F40.

First author partially supported by NSF Mathematical Sciences Postdoctoral Research Fellowship. 
A function $F: \mathcal{H}_{g} \rightarrow \mathbb{C}$ is called a modular form of weight $k$ with respect to $\Gamma \subset \Gamma_{g}$ if

$$
F(\gamma \tau)=\operatorname{det}(c \tau+d)^{k} F(\tau), \quad \forall \gamma=\left(\begin{array}{ll}
a & b \\
c & d
\end{array}\right) \in \Gamma, \forall \tau \in \mathcal{H}_{g}
$$

More generally, let $\rho: \mathrm{GL}(g, \mathbb{C}) \rightarrow$ End $V$ be some representation. Then a map $F: \mathcal{H}_{g} \rightarrow V$ is called a $\rho$ - or $V$-valued modular form, or simply a vector-valued modular form, if the choice of $\rho$ is clear, with respect to $\Gamma \subset \Gamma_{g}$ if

$$
F(\gamma \tau)=\rho(c \tau+d) F(\tau), \quad \forall \gamma=\left(\begin{array}{ll}
a & b \\
c & d
\end{array}\right) \in \Gamma, \forall \tau \in \mathcal{H}_{g}
$$

For $\varepsilon, \delta \in \mathbb{Z}_{2}^{g}$, thought of as vectors of zeros and ones, and $z \in \mathbb{C}^{g}$ we define the theta function with characteristic $[\varepsilon, \delta]$ to be

$$
\theta\left[\begin{array}{l}
\varepsilon \\
\delta
\end{array}\right](\tau, z):=\sum_{m \in \mathbb{Z}^{g}} \exp \pi i\left[\left(m+\frac{\varepsilon}{2}, \tau\left(m+\frac{\varepsilon}{2}\right)\right)+2\left(m+\frac{\varepsilon}{2}, z+\frac{\delta}{2}\right)\right] .
$$

A characteristic $[\varepsilon, \delta]$ is called even or odd depending on whether the scalar product $\varepsilon \cdot \delta \in \mathbb{Z}_{2}$ is zero or one, and the corresponding theta function is even or odd in $z$, respectively. The number of even (resp. odd) characteristics is $2^{g-1}\left(2^{g}+1\right)$ (resp. $\left.2^{g-1}\left(2^{g}-1\right)\right)$.

For $\varepsilon \in \mathbb{Z}_{2}^{g}$ we also define the second order theta function with characteristic $\varepsilon$ to be

$$
\Theta[\varepsilon](\tau, z):=\theta\left[\begin{array}{l}
\varepsilon \\
0
\end{array}\right](2 \tau, 2 z)
$$

The group $\Gamma_{g}$ acts on the set of characteristics as follows:

$$
\gamma\left(\begin{array}{l}
\varepsilon \\
\delta
\end{array}\right):=\left(\begin{array}{cc}
d & -c \\
-b & a
\end{array}\right)\left(\begin{array}{l}
\varepsilon \\
\delta
\end{array}\right)+\frac{1}{2}\left(\begin{array}{l}
\operatorname{diag}\left(c d^{t}\right) \\
\operatorname{diag}\left(a b^{t}\right)
\end{array}\right)
$$

where the resulting characteristics is taken modulo 2. This action is not transitive, in fact the parity of the characteristics is an invariant. The transformation law for theta functions under the action of the symplectic group is (see $\operatorname{Ig} 72$ ):

$$
\theta\left[\gamma\left(\begin{array}{l}
\varepsilon \\
\delta
\end{array}\right)\right]\left(\gamma \tau,(c \tau+d)^{-t} z\right)=\phi(\varepsilon, \delta, \gamma, \tau, z) \operatorname{det}(c \tau+d)^{1 / 2} \theta\left[\begin{array}{l}
\varepsilon \\
\delta
\end{array}\right](\tau, z),
$$

where $\phi$ is some complicated explicit function. It is further known (see Ig72, SM94]) that $\left.\phi\right|_{z=0}$ does not depend on $\tau$, and that for $\gamma \in \Gamma_{g}(4,8)$ we have $\left.\phi\right|_{z=0}=1$, while $\gamma \in \Gamma_{g}(4,8)$ acts trivially on the characteristics $[\varepsilon, \delta]$. Thus the values of theta functions at $z=$ 0 , called theta constants, are modular forms of weight one half with respect to $\Gamma_{g}(4,8)$. Similarly it is known that the theta constants of 
second order are modular forms of weight one half with respect to $\Gamma_{g}(2,4)$. The action of $\Gamma_{g}(2) / \Gamma_{g}(4,8)$ on the set of theta constants with characteristics is by certain characters whose values are fourth roots of the unity, and is well understood - see [SM94]. The action of $\Gamma_{g} / \Gamma_{g}(2)$ on the set of characteristics is by permutations.

All odd theta constants with characteristics vanish identically, as the corresponding theta functions are odd functions of $z$, and thus there are $2^{g-1}\left(2^{g}+1\right)$ non-trivial theta constants with characteristics, and $2^{g}$ theta constants of the second order.

Differentiating the theta transformation law above with respect to $z_{i}$ and then evaluating at $z=0$, we see that for $\gamma \in \Gamma_{g}(4,8)$ and $[\varepsilon, \delta]$ odd

$$
\left.\frac{\partial}{\partial z_{i}} \theta\left[\begin{array}{l}
\varepsilon \\
\delta
\end{array}\right](\tau, z)\right|_{z=0}=\left.\operatorname{det}(c \tau+d)^{1 / 2} \sum_{j}(c \tau+d)_{i j} \frac{\partial}{\partial z_{j}} \theta\left[\begin{array}{l}
\varepsilon \\
\delta
\end{array}\right](\gamma \tau, z)\right|_{z=0}
$$

in other words the gradient vector $\left\{\frac{\partial}{\partial z_{i}} \theta\left[\begin{array}{l}\varepsilon \\ \delta\end{array}\right](\tau, 0)\right\}_{\text {all } i}$ is a $\mathbb{C}^{g}$-valued modular form with respect to $\Gamma_{g}(4,8)$ under the representation $\rho(M):=$ $(\operatorname{det} M)^{1 / 2} \cdot M$.

The set of all even theta constants with characteristics defines the map

$$
\mathbb{P} T h: \mathcal{A}_{g}^{4,8} \rightarrow \mathbb{P}^{2^{g-1}\left(2^{g}+1\right)-1}, \quad \mathbb{P} T h(\tau):=\left\{\theta\left[\begin{array}{l}
\varepsilon \\
\delta
\end{array}\right](\tau, 0)\right\}_{\text {all even }[\varepsilon, \delta]}
$$

Theta constants of the second order similarly define the map

$$
\mathbb{P T} h_{2}: \mathcal{A}_{g}^{2,4} \rightarrow \mathbb{P}^{2^{g}-1}, \quad \mathbb{P} T h_{2}(\tau):=\{\Theta[\varepsilon](\tau, 0)\}_{\text {all } \varepsilon} .
$$

Considering the set of gradients of all odd theta functions at zero gives the map

$$
\operatorname{grTh}: \mathcal{H}_{g} \rightarrow\left(\mathbb{C}^{g}\right)^{\times 2^{g-1}\left(2^{g}-1\right)} \operatorname{grTh}(\tau):=\left\{\operatorname{grad}_{z} \theta\left[\begin{array}{l}
\varepsilon \\
\delta
\end{array}\right]\right\}_{\text {all odd }[\varepsilon, \delta]},
$$

which due to modular properties descends to the quotient map

$$
\operatorname{PgrTh}: \mathcal{A}_{g}^{4,8} \rightarrow\left(\mathbb{C}^{g}\right)^{\times 2^{g-1}\left(2^{g}-1\right)} / \rho(\mathrm{GL}(g, \mathbb{C})),
$$

where $\mathrm{GL}(g, \mathbb{C})$ acts simultaneously on all $\mathbb{C}^{g}$ 's in the product by $\rho$.

Because of Lefschetz theorem for abelian varieties for any $\tau$ the rank of the $2^{g-1}\left(2^{g}-1\right) \times g$ matrix of derivatives $\frac{\partial}{\partial_{z_{i}}} \theta\left[\begin{array}{l}\varepsilon \\ \delta\end{array}\right](\tau, 0)$ is always $g$ (see SM83]). Thus if we think of this matrix as a $g$-tuple of vectors in 
$\mathbb{C}^{2^{g-1}\left(2^{g}-1\right)}$, it is always non-degenerate. Thus the image of $\mathbb{P} g r T h$ in fact lies in the grassmannian,

$$
\operatorname{PgrTh}: \mathcal{A}_{g}^{4,8} \rightarrow \operatorname{Gr}_{\mathbb{C}}\left(g, 2^{g-1}\left(2^{g}-1\right)\right)
$$

of $g$-dimensional subspaces in $\mathbb{C}^{2^{g-1}\left(2^{g}-1\right)}$. The Plücker's coordinates of this map are modular forms of weight $\frac{g}{2}+1$ and have been extensively studied - see [Fr885], Fa79, [Ig80, SM83.

It is known that the map $\mathbb{P} T h$ is an embedding — see $\operatorname{Ig} 72$ and references therein. In SM94 it is shown that $\mathbb{P} T h_{2}$ is also injective. However, there appears to be a small gap in the proof there, so at the moment we can only say that the map is injective for $g \leq 3$ and generically injective for $g \geq 4$. In this work we will avoid using this result except for the case $g=2$. We remark that, because of the transformation formula of theta functions, all the above maps are $\Gamma_{g^{-}}$ equivariant. Here we prove the following properties of $\mathbb{P} g r T h$ :

Theorem 1. For $g \geq 3$ the map $\mathbb{P}$ grTh is generically injective on $\mathcal{A}_{g}^{4,8}$. For genus 2 the map is finite of degree 16.

In the course of the proof we give explicitly an open set in $\mathcal{A}_{g}^{4,8}$ where the map is injective, and obtain, denoting by $\mathcal{J}_{g} \subset \mathcal{A}_{g}$ the locus of Jacobians,

Corollary 2. For $g \geq 3$ the map $\mathbb{P}$ grTh is also generically injective on $\mathcal{J}_{g}^{4,8}$.

We will also show

Theorem 3. For $g \geq 2$ the map $\mathbb{P}$ grTh is injective on tangent spaces.

Remark 4. The map PgrTh is closely related to the indicated in CS00 generalization to abelian varieties of the map obtained by sending a Jacobian to the collection of the hyperplanes tangent to the canonical curve at $g-1$ points, considered in [Ca01, CSO0, CS02]. This map itself is in fact the generalization of the map of a plane quartic to the set of its bitangents. It was shown by Aronhold in Ar872 that some subset of bitangents (i.e. their directions), now called an Aronhold system, together with the points of their tangency serves to recover the curve, but until CS00 it was not known that the bitangent directions themselves determine a (generic) curve.

Indeed, suppose we have a hyperplane tangent to the canonical curve at $g-1$ points. The reduced divisor that it cuts on the curve is then the square root of the canonical, and thus a theta characteristic. However, it is an effective theta characteristic, which for a generic curve means it is an odd theta characteristic. On the other hand, for each odd theta 
characteristics we get a $(g-1)$-tangent hyperplane to the canonical curve, and analytically it is clear what the direction of this hyperplane is: it is given by the gradient of the corresponding odd theta function with characteristics at zero.

In our terms the map to $(g-1)$-tangent hyperplanes is thus the map

$$
\operatorname{gr} \mathbb{P} T h: \mathcal{J}_{g} \rightarrow S^{2^{g-1}\left(2^{g}-1\right)}\left(\mathbb{P}^{g-1}\right) / \operatorname{PGL}(g, \mathbb{C})
$$

of the locus of Jacobians $\mathcal{J}_{g}$, obtained by sending $\tau$ to the set of gradients of all odd theta constants, but each projectivized independently, and considered as a point in $\mathbb{P}^{g-1}$ and not in $\mathbb{C}^{g}$ (we have the symmetric power of $\mathbb{P}^{g-1}$ here instead of the direct power because we are forgetting the level structure, and thus the characteristics may be permuted).

This map gr $\mathbb{P} T h$ considered by Caporaso and Sernesi is quite different from our $\mathbb{P} g r T h$. As explained in [CS00] and [CS02], grPTh is not defined on all of $\mathcal{J}_{g}$ or $\mathcal{A}_{g}$ - the problem occurs for those $\tau$ for which one of the components of $\operatorname{grTh}(\tau)$ is equal to zero. Where it is defined, it factors through $\mathbb{P} g r T h$ :

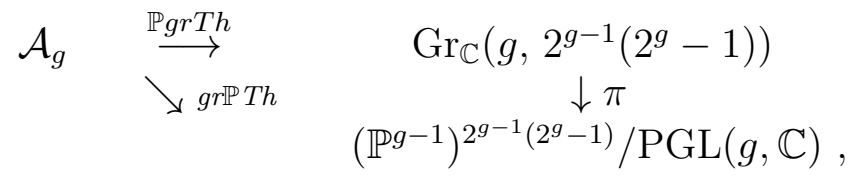

where $\pi$ is the natural projection, which is not always defined.

In CS00 and CS02 it is shown that when restricted to its domain on the Jacobian locus, gr $\mathbb{P} T h$ is generically injective. While our corollary 2 states that $\left.\mathbb{P} g r T h\right|_{\mathcal{J}_{g}}$ is generically injective, it does not serve to reproduce the result of Caporaso and Sernesi, as we do not handle the projectivization map $\pi$, which might collapse images different points.

In the following, we will often omit the arguments $\tau$ and $z=0$ for theta functions and their derivatives, and will write $\partial_{z_{i}}$ instead of $\frac{\partial}{\partial z_{i}}$. The letters of the Latin alphabet will denote coordinates for vectors in $\mathbb{C}^{g}$, i.e. will range from 1 to $g$. The letters of the Greek alphabet denote characteristics for $g$-dimensional theta functions, i.e. lie in $\mathbb{Z}_{2}^{g}$.

\section{2. $\theta$ 'S AND $\Theta '$ 'S}

The fundamental relation between theta functions with characteristics and theta functions of the second order comes from the fact that the squares of theta functions with characteristics are sections of the bundle on the abelian variety for which the theta functions of thesecond order form a basis for the space of sections. The relationship and others more general are special cases of Riemann's addition theorem 
for theta functions (see, for example, $\operatorname{Ig} 72)$ :

$$
\begin{gathered}
\theta\left[\begin{array}{l}
\alpha \\
\beta
\end{array}\right](2 \tau, 2 z) \theta\left[\begin{array}{c}
\alpha+\varepsilon \\
\beta
\end{array}\right](2 \tau, 2 x) \\
=\frac{1}{2^{g}} \sum_{\sigma \in \mathbb{Z}_{2}^{g}}(-1)^{\alpha \cdot \sigma} \theta\left[\begin{array}{c}
\varepsilon \\
\beta+\sigma
\end{array}\right](\tau, z+x) \theta\left[\begin{array}{l}
\varepsilon \\
\sigma
\end{array}\right](\tau, z-x),
\end{gathered}
$$

which is valid for all $\tau, z, x$ and $\alpha, \beta, \varepsilon$. Let us denote

$$
\begin{gathered}
C_{i j \varepsilon \delta}^{\beta}(\tau):=\partial_{z_{i}} \theta\left[\begin{array}{c}
\varepsilon \\
\beta+\delta
\end{array}\right](\tau, 0) \partial_{z_{j}} \theta\left[\begin{array}{l}
\varepsilon \\
\delta
\end{array}\right](\tau, 0) \\
+\partial_{z_{j}} \theta\left[\begin{array}{c}
\varepsilon \\
\beta+\delta
\end{array}\right](\tau, 0) \partial_{z_{i}} \theta\left[\begin{array}{l}
\varepsilon \\
\delta
\end{array}\right](\tau, 0),
\end{gathered}
$$

which is zero unless $[\varepsilon, \beta+\delta]$ and $[\varepsilon, \delta]$ are odd, and

$$
\begin{gathered}
A_{i j \varepsilon \delta}^{\beta}(\tau):=\partial_{z_{i}} \partial_{z_{j}} \theta\left[\begin{array}{l}
\delta \\
\beta
\end{array}\right](2 \tau, 0) \theta\left[\begin{array}{l}
\varepsilon \\
\beta
\end{array}\right](2 \tau, 0)- \\
\theta\left[\begin{array}{l}
\delta \\
\beta
\end{array}\right](2 \tau, 0) \partial_{z_{i}} \partial_{z_{j}} \theta\left[\begin{array}{l}
\varepsilon \\
\beta
\end{array}\right](2 \tau, 0),
\end{gathered}
$$

which is zero unless $[\delta, \beta]$ and $[\varepsilon, \beta]$ are both even characteristics. Note also that by the heat equation we have $\partial_{z_{i}} \partial_{z_{j}} \theta=\partial_{\tau_{i j}} \theta$ up to a constant that is not important to us.

We then have the following relation

Lemma 5. If $[\varepsilon, \delta]$ and $[\varepsilon, \beta+\delta]$ are odd characteristics,

$$
C_{i j \varepsilon \delta}^{\beta}=\frac{1}{2} \sum_{\alpha \in \mathbb{Z}_{2}^{g}}(-1)^{\alpha \cdot \delta} A_{i j \varepsilon+\alpha \alpha}^{\beta} .
$$

Proof. Let us take the sum of the equations (3) for different $\alpha$, each with coefficient $(-1)^{\alpha \cdot \delta}$, where $\delta$ is some characteristic. We get

$$
\begin{gathered}
\sum_{\alpha \in \mathbb{Z}_{2}^{g}}(-1)^{\alpha \cdot \delta} \theta\left[\begin{array}{c}
\alpha \\
\beta
\end{array}\right](2 \tau, 2 z) \theta\left[\begin{array}{c}
\alpha+\varepsilon \\
\beta
\end{array}\right](2 \tau, 0) \\
=\frac{1}{2^{g}} \sum_{\alpha, \sigma \in \mathbb{Z}_{2}^{g}}(-1)^{\alpha \cdot(\sigma+\delta)} \theta\left[\begin{array}{c}
\varepsilon \\
\beta+\sigma
\end{array}\right](\tau, z) \theta\left[\begin{array}{l}
\varepsilon \\
\sigma
\end{array}\right](\tau, z) \\
=\theta\left[\begin{array}{c}
\varepsilon \\
\beta+\delta
\end{array}\right](\tau, z) \theta\left[\begin{array}{l}
\varepsilon \\
\delta
\end{array}\right](\tau, z) .
\end{gathered}
$$

Now differentiate this relation twice with respect to $z_{i}$ and $z_{j}$ and then evaluate at $z=0$ to prove the lemma.

Moreover, the expression of $C$ 's in terms of $A$ 's is invertible: 


\section{Lemma 6.}

$$
A_{i j \alpha+\varepsilon \alpha}^{\beta}=\frac{1}{2^{g-1}} \sum_{\{\sigma \mid[\varepsilon, \sigma] \text { odd }\}}(-1)^{\alpha \cdot \sigma} C_{i j \varepsilon \sigma}^{\beta}
$$

Proof. In (3), we assume that $[\alpha, \beta]$ and $[\alpha+\varepsilon, \beta]$ are even characteristics. Differentiating, we get

$$
\begin{gathered}
\partial_{z_{i}} \partial_{z_{j}} \theta\left[\begin{array}{l}
\alpha \\
\beta
\end{array}\right](2 \tau, 0) \theta\left[\begin{array}{c}
\alpha+\varepsilon \\
\beta
\end{array}\right](2 \tau, 0) \\
=\left.\frac{1}{2^{g}} \partial_{z_{i}} \partial_{z_{j}}\left(\sum_{\sigma \in \mathbb{Z}_{2}^{g}}(-1)^{\alpha \cdot \sigma} \theta\left[\begin{array}{c}
\varepsilon \\
\beta+\sigma
\end{array}\right](\tau, z) \theta\left[\begin{array}{l}
\varepsilon \\
\sigma
\end{array}\right](\tau, z)\right)\right|_{z=0} .
\end{gathered}
$$

Similarly, switching $\alpha$ and $\alpha+\varepsilon$ we have

$$
\begin{gathered}
\theta\left[\begin{array}{l}
\alpha \\
\beta
\end{array}\right](2 \tau, 0) \partial_{z_{i}} \partial_{z_{j}} \theta\left[\begin{array}{c}
\alpha+\varepsilon \\
\beta
\end{array}\right](2 \tau, 0) \\
\left.=\frac{1}{2^{g}} \partial_{z_{i}} \partial_{z_{j}}\left(\sum_{\sigma \in \mathbb{Z}_{2}^{g}}(-1)^{(\alpha+\varepsilon) \cdot \sigma} \theta\left[\begin{array}{c}
\varepsilon \\
\beta+\sigma
\end{array}\right](\tau, z) \theta\left[\begin{array}{l}
\varepsilon \\
\sigma
\end{array}\right](\tau, z)\right)\right)\left.\right|_{z=0} .
\end{gathered}
$$

Subtracting and computing separately for the cases of $[\varepsilon, \sigma]$ odd and even, we get the statement of the lemma.

\section{Recovering $\mathbb{P} T h_{2}(\tau)$ FROM $\operatorname{PgrTh}(\tau)$}

Proposition 7. The following identity holds for all $i, j, \varepsilon, \delta, \sigma, \beta$, and all $\tau \in \mathcal{H}_{g}:$

$$
A_{i j, \delta \delta}^{\beta}(\tau) \theta\left[\begin{array}{l}
\sigma \\
\beta
\end{array}\right](2 \tau)+A_{i j, \delta \sigma}^{\beta}(\tau) \theta\left[\begin{array}{l}
\varepsilon \\
\beta
\end{array}\right](2 \tau)+A_{i j, \sigma \varepsilon}^{\beta}(\tau) \theta\left[\begin{array}{l}
\delta \\
\beta
\end{array}\right](2 \tau)=0
$$

Proof. From the definition of $A$ 's it follows that the above expression

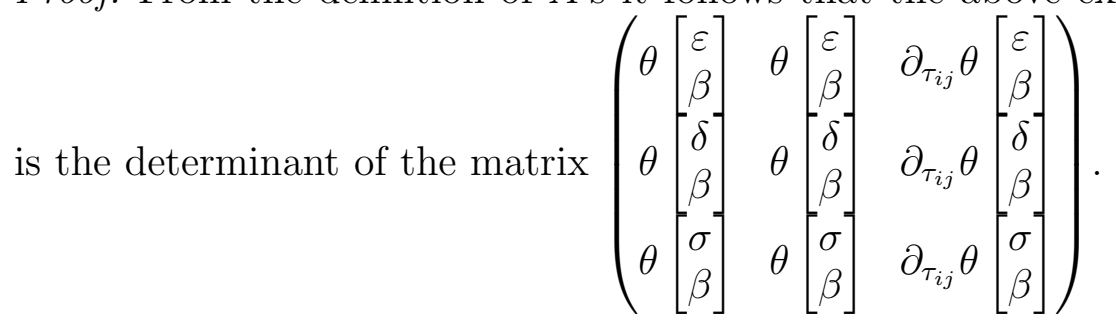

Proposition 8. For any $i, j, I, J, \varepsilon, \delta, \sigma, \tau$ we have

$$
\begin{aligned}
& \left(A_{i j, \delta \delta}^{\beta}(\tau) A_{I J, \sigma \delta}^{\beta}(\tau)-A_{I J, \varepsilon \delta}^{\beta}(\tau) A_{i j, \sigma \delta}^{\beta}(\tau)\right) \theta\left[\begin{array}{l}
\varepsilon \\
\beta
\end{array}\right](2 \tau) \\
= & \left(A_{i j, \delta \varepsilon}^{\beta}(\tau) A_{I J, \sigma \varepsilon}^{\beta}(\tau)-A_{I J, \delta \varepsilon}^{\beta}(\tau) A_{i j, \sigma \varepsilon}^{\beta}(\tau)\right) \theta\left[\begin{array}{l}
\delta \\
\beta
\end{array}\right](2 \tau) .
\end{aligned}
$$


Proof. The identity is straightforward if we substitute the definition of $A$ 's in terms of $\theta$ 's and their derivatives. Indeed

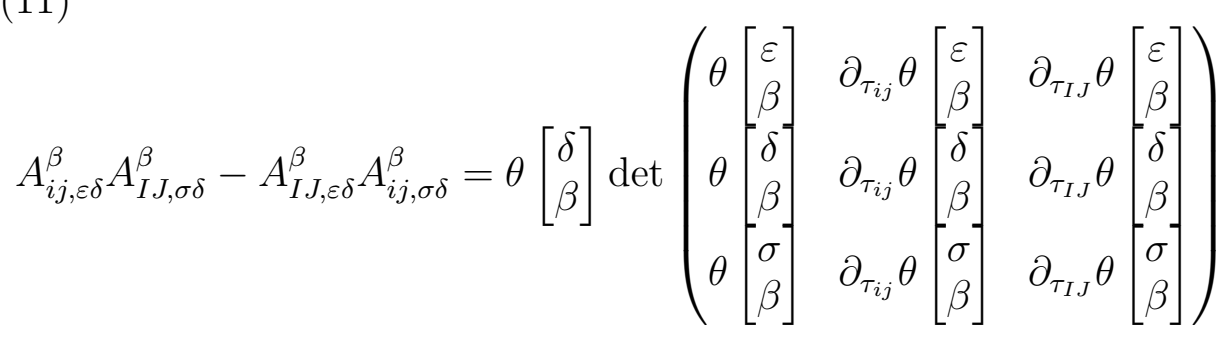

evaluated at $2 \tau$. Another proof would be to use the previous proposition for $\varepsilon, \delta, \sigma$ with $i, j$ and then with $I, J$ to express $\theta\left[\begin{array}{l}\sigma \\ \beta\end{array}\right]$ in two different ways, and then equate these two expressions.

Let us consider the case $\beta=0$ for the propositions above. Then the $A_{i j, \varepsilon \delta}^{0}$ are $2 \times 2$ minors of the $2^{g} \times\left(\frac{g(g+1)}{2}+1\right)$ matrix $M$ with columns $\left(\Theta[\varepsilon],\left\{\partial_{\tau_{i j}} \Theta[\varepsilon]\right\}_{i \leq j}\right)_{\text {all i,j }}$ (i.e. in each column there are the values either of $\Theta[\varepsilon]$ or of its derivative, for all $\varepsilon \in \mathbb{Z}_{2}^{g}$ ). This has maximal rank, equal to $\frac{g(g+1)}{2}+1$, for irreducible abelian varieties — see Sa83 and SM94. Inductively for reducible abelian varieties we see that for $g \geq 2$ the rank of $M$ is at least three.

Proposition 9. For $\beta=0$ the system of equations (7) has a unique projective solution for $\{\Theta[\varepsilon](\tau)\}_{\text {all } \varepsilon}$ for fixed $A$ 's. Since $A$ 's are expressible in terms of $C$ 's, which are combinations of gradients of odd theta functions, this means that $\mathbb{P} T h_{2}(\tau)$ is determined uniquely by $\mathbb{P g r T h}(\tau)$.

Proof. This is a consequence of the fact that the matrix $M$ above has at least three, and that the solutions of the system (17) are invariant under the action of $\operatorname{GL}(g, \mathbb{C})$. Basically we need to show that the system has maximal rank. Suppose we are given $\mathbb{P} g r T h(\tau)$, i.e. all $A_{i j, \varepsilon \delta}^{0}$ 's. Let us pick a representative $\tau \in \mathcal{H}_{g}$ and think of $\operatorname{gr} T h(\tau)$ - we will deal with the action of $\mathrm{GL}(g, \mathbb{C})$ later.

Since the matrix $M$ described above has rank at least 3, we can pick a non-degenerate $3 \times 3$ minor in it. For irreducible $\tau$ the matrix $M$ is of maximal rank, and thus this minor can be chosen to contain the first column. From the fact that the theta constants of reducible abelian varieties are products of lower-dimensional theta constants it follows that such a choice is also possible for reducible abelian varieties. 
Suppose now that this non-degenerate minor is

$$
\operatorname{det}\left(\begin{array}{ccc}
\Theta[\varepsilon] & \partial_{\tau_{i j}} \Theta[\varepsilon] & \partial_{\tau_{I J}} \Theta[\varepsilon] \\
\Theta[\delta] & \partial_{\tau_{i j}} \Theta[\delta] & \partial_{\tau_{I J}} \Theta[\delta] \\
\Theta[\sigma] & \partial_{\tau_{i j}} \Theta[\sigma] & \partial_{\tau_{I J}} \Theta[\sigma]
\end{array}\right) \neq 0 .
$$

Then at least one of $\Theta[\varepsilon], \Theta[\delta], \Theta[\sigma]$ must also be non-zero — by renaming let it be $\Theta[\varepsilon]$. Then the combination of $A$ 's in the right-hand-side of the formula (11) is non-zero, and thus we can use proposition 8 to express $\frac{\Theta[\delta]}{\Theta[\varepsilon]}$ and $\frac{\Theta[\sigma]}{\Theta[\varepsilon]}$ in terms of $A$ 's, i.e. in terms of $\operatorname{gr} T h(\tau)$.

Furthermore, since $\Theta[\varepsilon]$ and the $3 \times 3$ minor above are non-zero, some $2 \times 2$ subminor containing $\Theta[\varepsilon]$ must also be non-zero. By renaming let it be

$$
\operatorname{det}\left(\begin{array}{cc}
\Theta[\varepsilon] & \partial_{\tau_{i j}} \Theta[\varepsilon] \\
\Theta[\delta] & \partial_{\tau_{i j}} \Theta[\delta]
\end{array}\right)=A_{i j, \varepsilon \delta}^{0} \neq 0
$$

Then we can use proposition[7]with $\varepsilon, \delta, i, j$ and any $\sigma$ to express all $\frac{\Theta[\sigma]}{\Theta[\varepsilon]}$ in terms of $A$ 's. Thus from $g r T h(\tau)$ we can recover $\mathbb{P} T h_{2}(\tau)$ uniquely.

Now we have to deal with the action of $\operatorname{GL}(g, \mathbb{C})$ to finish the proof. However, the system (9) is acted upon by the adjoint action of $\mathrm{GL}(g, \mathbb{C})$ (if we consider each $A_{i j \varepsilon \delta}^{0}$ as a matrix labeled by $i, j$ ), and thus transformed into an equivalent system. This equivalent system will have the same solutions, and as we have shown the solution to be unique, it has the same solution.

\section{Generic injectivity of $\mathbb{P} g r T h$}

If it were known that $\mathbb{P} T h_{2}$ is injective, we would be already done, and could conclude the injectivity of $\mathbb{P} g r T h$ at level $(2,4)$. But, as this is not yet known, we need to do extra work.

Remark 10. The genus $g=2$ case is rather special: in this case it is known that $\mathbb{P T h} h_{2}$ is injective; however, there are only six odd characteristics, while

$$
\left|\Gamma_{2}(2,4) / \Gamma_{2}(4,8)\right|=2^{10},
$$

Thus in genus two the map $\mathbb{P} g r T h(\tau)$ factors over some subgroup $\Gamma$ such that $\Gamma_{2}(4,8) \subset \Gamma \subset \Gamma_{2}(2,4)$, and the injectivity of $\mathbb{P} g r T h$ holds only on $\mathcal{A}_{2}^{\Gamma}$.

In general, to recover $\mathbb{P} T h(\tau)$ from $\mathbb{P} T h_{2}(\tau)$, we just need to know which sign to choose for each $\theta\left[\begin{array}{l}\varepsilon \\ \delta\end{array}\right]$, since their squares are already expressible in terms of theta constants of the second order by (17). 
To get more control over the signs, let us consider the case $\beta \neq 0$ of the equations (3). Then $A_{i j, \varepsilon \delta}^{\beta}$ are $2 \times 2$ minors of the $2^{g} \times\left(\frac{g(g+1)}{2}+1\right)$ matrix $M^{\beta}$ consisting of $\left(\theta\left[\begin{array}{l}\alpha \\ \beta\end{array}\right](2 \tau, 0),\left\{\partial_{\tau_{i j}} \theta\left[\begin{array}{l}\alpha \\ \beta\end{array}\right](2 \tau, 0)\right\}_{i \leq j}\right)_{\text {all } \alpha}$, which, unlike the $M$ considered above, has $2^{g-1}$ null rows corresponding to those $\alpha$ for which $[\alpha, \beta]$ is odd. To use an argument similar to the one in the previous section relating $\mathbb{P} g r T h$ and $\mathbb{P} T h_{2}$, we need

Lemma 11. For $g \geq 3$ the matrix $M^{\beta}$ has rank at least three for all $\tau$.

Proof. Fix an irreducible period matrix $\tau$ and consider the abelian variety $X:=X_{\tau}$. Let $\mathcal{L}$ denote the symmetric line bundle inducing a principal polarization on $X$, for which $\theta\left[\begin{array}{l}0 \\ 0\end{array}\right](\tau, z)$ is the basis for sections. We denote by $\Theta$ the associated divisor. For any $x \in X$, let $t_{x}: X \rightarrow X$ be the traslation by $x$. Setting $x:=\beta / 4$, we consider the line bundle $\mathcal{N}:=t_{x}^{*} \mathcal{L}^{2}$. A basis for the sections of $\mathcal{N}$ are the theta functions $\theta\left[\begin{array}{l}\alpha \\ \beta\end{array}\right](2 \tau, 2 z)$. As a consequence of (프), we see that for any $x \in X$, all

$$
\theta\left[\begin{array}{l}
0 \\
\beta
\end{array}\right](\tau, z+x) \theta\left[\begin{array}{l}
0 \\
0
\end{array}\right](\tau, z-x)
$$

are linear combinations of $\theta\left[\begin{array}{l}\alpha \\ \beta\end{array}\right](2 \tau, 2 z)$. We recall that if $\tau$ is irreducibile, the Gauss maps $G_{x}: t_{x}^{*} \Theta \rightarrow \mathbb{P}^{g-1}$ are dominant.

Suppose $M^{\beta}$ is not of maximal rank. Then for some $\lambda$ and $c$ 's we have

$$
\sum_{i \leq j} c_{i j} \partial_{\tau_{i j}} \theta\left[\begin{array}{l}
\alpha \\
\beta
\end{array}\right](2 \tau, 0)=\lambda \theta\left[\begin{array}{l}
\alpha \\
\beta
\end{array}\right](2 \tau, 0)
$$

Thus we have

$$
\begin{gathered}
\left.\sum_{i \leq j} c_{i j} \partial_{z_{i}} \partial_{z_{j}}\left(\theta\left[\begin{array}{l}
0 \\
\beta
\end{array}\right](\tau, z-x) \theta\left[\begin{array}{l}
0 \\
0
\end{array}\right](\tau, z+x)\right)\right|_{z=0} \\
=\lambda \theta\left[\begin{array}{l}
0 \\
\beta
\end{array}\right](\tau, x) \theta\left[\begin{array}{l}
0 \\
0
\end{array}\right](\tau, x)
\end{gathered}
$$

If $x$ does not belong to $\Theta \cup t_{\beta / 2}^{*} \Theta$, the coefficient of $\lambda$ in the above relation is not zero. Vice versa, assuming that $x \in \Theta \cap t_{\beta / 2}^{*} \Theta$, we have

$$
\sum_{i \leq j} c_{i j} \partial_{z_{i}} \theta\left[\begin{array}{l}
0 \\
\beta
\end{array}\right](\tau, x) \partial_{z_{j}} \theta\left[\begin{array}{l}
0 \\
0
\end{array}\right](\tau, x)+\partial_{z_{j}} \theta\left[\begin{array}{l}
0 \\
\beta
\end{array}\right](\tau, x) \partial_{z_{i}} \theta\left[\begin{array}{l}
0 \\
0
\end{array}\right](\tau, x)=0
$$


Now, since $\tau$ is irreducible, the singular locus of $\Theta$ has codimension at least two in $\Theta$, see [EL97], so there exists such an $x$ in the smooth part of $\Theta$ and $t_{\beta / 2}^{*} \Theta$. Moreover, for $g \geq 3$ by a linear transformation we can find $x_{1}$ and $x_{2}$ such that

$$
\partial_{z_{j}} \theta\left[\begin{array}{l}
0 \\
0
\end{array}\right]\left(\tau, x_{1}\right)=\delta_{j}^{1} \text { and } \partial_{z_{j}} \theta\left[\begin{array}{l}
0 \\
0
\end{array}\right]\left(\tau, x_{2}\right)=\delta_{j}^{2},
$$

where $\delta_{i}^{j}$ is Krönecker's delta, and either $\partial_{z_{j}} \theta\left[\begin{array}{l}0 \\ \beta\end{array}\right]\left(\tau, x_{2}\right)$ is not proportional to $\partial_{z_{j}} \theta\left[\begin{array}{l}0 \\ 0\end{array}\right]\left(\tau, x_{1}\right)$ or $\partial_{z_{j}} \theta\left[\begin{array}{l}0 \\ \beta\end{array}\right]\left(\tau, x_{1}\right)$ is not proportional to $\partial_{z_{j}} \theta\left[\begin{array}{l}0 \\ 0\end{array}\right]\left(\tau, x_{2}\right)$. These properties impose three linearly independent conditions on the coefficients $c_{i j}, \lambda$. Hence, for irreducible $\tau$ the matrix $M^{\beta}$ has at least three linearly independent columns. If the point $\tau$ is reducible, we can use similar facts about the Gauss map, do directly the genus three case and use some inductive argument to finish the proof.

Thus we get a generalization of proposition 9 in the same way:

Proposition 12. Given $\mathbb{P} g r T h(\tau)$, the equations (9) for $\beta \neq 0$ have a unique projective solution for $\left\{\theta\left[\begin{array}{l}\alpha \\ \beta\end{array}\right](2 \tau, 0)\right\}_{\text {all } \alpha}$.

From the above proposition and formula (7) it follows that

Corollary 13. All products of the type

$$
\theta\left[\begin{array}{c}
\varepsilon \\
\beta+\delta
\end{array}\right](\tau, 0) \theta\left[\begin{array}{l}
\varepsilon \\
\delta
\end{array}\right](\tau, 0)
$$

are determined by $\mathbb{P}$ grTh uniquely up to a multiplicative constant $t_{[0, \beta]}$.

Obviously the above statement is true for every point $\tau$, thus, using the $\Gamma_{g}$-equivariance of the map $\mathbb{P} g r T h$ and observing that the homogenuos action of $\Gamma_{g}$ on the set of characteristics is transitive on the set of characteristics different from $[0,0]$, by using the above corollary stated for points $\gamma \tau$ for all $\gamma$ we prove

Corollary 14. All products of the type

$$
\theta\left[\begin{array}{l}
\alpha \\
\beta
\end{array}\right](\tau, 0) \theta\left[\begin{array}{l}
\alpha+\varepsilon \\
\beta+\delta
\end{array}\right](\tau, 0)
$$

are determined by $\mathbb{P} g r T h(\tau)$ uniquely up to a multiplicative constant $t_{\varepsilon, \delta}$. 
Now we are able to prove our main theorem.

Proof of theorem 1. We will show that generically $\mathbb{P} g r T h(\tau)$ determines $\mathbb{P} T h(\tau)$ uniquely. Assume that there are two points $\tau$ and $\tau^{\prime}$ for which $\mathbb{P} g r T h(\tau)=\mathbb{P} g r T h\left(\tau^{\prime}\right)$. Since $\mathbb{P} T h_{2}(\tau)=\mathbb{P} T h_{2}\left(\tau^{\prime}\right)$ by proposition 9.

$$
\theta\left[\begin{array}{l}
\alpha \\
\beta
\end{array}\right](\tau, 0)^{2}=c^{2} \theta\left[\begin{array}{l}
\alpha \\
\beta
\end{array}\right]\left(\tau^{\prime}, 0\right)^{2} \quad \forall[\alpha, \beta],
$$

where $c$ is a constant independent of $\alpha, \beta$. Hence

$$
\theta\left[\begin{array}{l}
\alpha \\
\beta
\end{array}\right](\tau, 0)=c s_{\alpha, \beta} \theta\left[\begin{array}{l}
\alpha \\
\beta
\end{array}\right]\left(\tau^{\prime}, 0\right),
$$

where $s_{\alpha, \beta}$ is a sign depending on $\alpha, \beta$. Replacing $\tau$ by $\gamma \tau$ and $c$ by $-c$ if necessary, we can assume $\theta\left[\begin{array}{l}0 \\ 0\end{array}\right](\tau, 0) \neq 0$ and $s_{0,0}=1$. Now from the two previous corollaries it follows that

$$
t_{\alpha, \beta}=t_{0,0} s_{\alpha, \beta}, \quad s_{\alpha, \beta} s_{\varepsilon, \delta}=s_{\alpha+\varepsilon, \beta+\delta} \quad \forall \alpha, \varepsilon, \delta, \beta
$$

whenever

$$
\theta\left[\begin{array}{l}
\alpha \\
\beta
\end{array}\right](\tau, 0) \cdot \theta\left[\begin{array}{l}
\varepsilon \\
\delta
\end{array}\right](\tau, 0) \cdot \theta\left[\begin{array}{l}
\alpha+\varepsilon \\
\beta+\delta
\end{array}\right](\tau, 0) \neq 0
$$

We would like to prove that all $s_{\alpha, \beta}=1$. Using (3) we can easily check that for all $[\varepsilon, \delta]$, among the products $\theta\left[\begin{array}{c}\alpha \\ \beta\end{array}\right](\tau, 0) \theta\left[\begin{array}{c}\alpha+\varepsilon \\ \beta+\delta\end{array}\right](\tau, 0)$ appearing in Corollary 14 there is at least one different from 0. Hence there is a linear basis $\left[\varepsilon_{1}, \delta_{1}\right], \ldots,\left[\varepsilon_{2 g}, \delta_{2 g}\right]$ for the set of characteristics such that the associated theta constants do not vanish at $\tau$. We denote by $A_{1}(\tau)$ this set of characteristics. Now recall that an element $\gamma=$ $\left(\begin{array}{ll}a & b \\ c & d\end{array}\right) \in \Gamma_{g}(4)$ acts on theta constants by the character

$$
\phi(\varepsilon, \delta, \gamma)=(-1)^{\frac{\operatorname{diag}(\mathrm{b}) \cdot \varepsilon}{4}}(-1)^{\frac{\operatorname{diag}(\mathrm{c}) \cdot \delta}{4}}
$$

by the theta transformation law (11). We observe that on one side this action is compatible with the statement of Corollary 14. Thus we can choose $\gamma \in \Gamma_{g}(4)$ such that

$$
\theta\left[\begin{array}{l}
\varepsilon_{j} \\
\delta_{j}
\end{array}\right](\tau, 0)=c \theta\left[\begin{array}{l}
\varepsilon_{j} \\
\delta_{j}
\end{array}\right]\left(\gamma \tau^{\prime}, 0\right),
$$

i.e. $s_{\varepsilon_{j}, \delta_{j}}=1$ for all $j=1, \ldots, 2 g$.

Now let $A(\tau)$ be the set of characteristics whose associated theta constants do not vanish at $\tau$. We denote by $A_{2}(\tau)$ the subset of $A(\tau)$ consisting of the elements of $A_{1}(\tau)$ and the sums of two elements of 
$A_{1}(\tau)$ that are still in $A(\tau)$. Since the condition (13) holds for characteristics in $A_{2}(\tau)$, (12) is satisfied and thus $s_{\varepsilon, \delta}=1$ since this is true for $A_{1}(\tau)$.

Furthermore, let us denote by $A_{3}(\tau)$ the subset of $A$ whose elements are either in $A_{2}(\tau)$ or sums of two elements of $A_{2}(\tau)$. Iterating the process, we get a certain subset $B(\tau)$. We would like to have $B(\tau)=$ $A(\tau)$. Obviously this holds and we have no trouble if we assume the non-vanishing of all theta constants with characteristic $[\varepsilon, 0]$ and $[0, \delta]$ : then whenever $\theta\left[\begin{array}{l}\varepsilon \\ \delta\end{array}\right](\tau, 0) \neq 0$, we can use

$$
\theta\left[\begin{array}{l}
\varepsilon \\
0
\end{array}\right](\tau, 0) \theta\left[\begin{array}{l}
0 \\
\delta
\end{array}\right](\tau, 0) \theta\left[\begin{array}{l}
\varepsilon \\
\delta
\end{array}\right](\tau, 0) \neq 0
$$

for the condition (13) and by formula (12) we are done. So if we restrict to the open set determined by this non-vanishing, we have $\mathbb{P} T h(\tau)=\mathbb{P} T h\left(\gamma \tau^{\prime}\right)$ for some $\gamma \in \Gamma_{g}$, and thus from the injectivity of $\mathbb{P} T h$ follows that $\tau$ and $\gamma \tau^{\prime}$ are $\Gamma_{g}(4,8)$-conjugate, so that also $\mathbb{P} g r T h(\tau)=\mathbb{P} g r T h\left(\gamma \tau^{\prime}\right)$. Now it is left to show that $\gamma \in \Gamma_{g}(4,8)$.

Assume the contrary: $\gamma \in \Gamma_{g}(4) \backslash \Gamma_{g}(4,8)$. We claim that then $\gamma$ acts non-trivially on $\mathbb{P} g r T h(\tau)$, so that we would have $\operatorname{PgrTh}(\tau)=$ $\mathbb{P} g r T h\left(\gamma \tau^{\prime}\right) \neq \mathbb{P} g r T h\left(\tau^{\prime}\right)$, which is a contradiction. Indeed, $\gamma$ acts on each gradient by multiplication by a sign $\phi(\varepsilon, \delta, \gamma)$. Consider all odd $[\varepsilon, \delta]$ such that $\phi(\varepsilon, \delta, \gamma)=-1$ : if for at least one of those and one of the remaining (otherwise there is multiplication by -1 ) the associated gradient $\operatorname{grad}_{z} \theta\left[\begin{array}{l}\varepsilon \\ \delta\end{array}\right]$ is not the zero vector, we are done. Up to conjugating by some element of $\Gamma_{g}$, we can assume that the $b$ in $\gamma$ is such that $\operatorname{diag}(b) \equiv 0 \bmod 8$. Since all level subgroups are normal, a conjugate of $\gamma$ lies exactly in the level subgroups in which $\gamma$ lies.

From formula (14) we see that if $\operatorname{diag} c \cdot \delta \equiv 4 \bmod 8$ for some fixed $\delta$ (resp. $\equiv 0)$, then $\phi(\varepsilon, \delta, \gamma)=-1$ (resp. $=1)$ for all $\varepsilon$. But since the map $X_{\tau} \rightarrow \mathbb{P}^{2^{g}-1}$ defined by $z \rightarrow \theta\left[\begin{array}{l}\varepsilon \\ 0\end{array}\right](\tau, z)$ is of maximal rank at the point $\delta / 2$ (recall that $\left.\theta\left[\begin{array}{l}\varepsilon \\ 0\end{array}\right](\tau, z+\delta / 2)=\theta\left[\begin{array}{l}\varepsilon \\ \delta\end{array}\right](\tau, z)\right)$, all gradients at zero of odd theta functions of the form $\theta\left[\begin{array}{l}\varepsilon \\ \delta\end{array}\right]$ cannot vanish simultaneously for all $\varepsilon$.

Remark 15. As a consequence of the above proof, if we have $B(\tau)=$ $A(\tau)$ for all $\tau$, the map $\mathbb{P} g r T h$ is injective. 
To the best of our knowledge this is always true. In particular it easy to check that even when $\tau$ is $\Gamma_{g}$-conjugate to a diagonal matrix, i.e. is the period matrix of a product of elliptic curves and thus has the maximal possible number of vanishing theta constants.

Thus we can say that $\mathbb{P} g r T h$ is injective, if for all $\tau$ the corresponding subset $A(\tau)$ contains $A(\tilde{\tau})$, with $\tilde{\tau}$ being $\Gamma_{g}$-conjugate to a diagonal matrix. For all examples of abelian varieties that we know the set of characteristics for which the associated theta constants vanish is always contained in such a set for a diagonal period matrix, up to conjugation. Thus in all the examples that we know we do have $A(\tau) \supset A(\tilde{\tau})$ and the map $\mathbb{P} g r T h$ is injective at $\tau$.

Since for $g=3,4$ the combinatorics of the possible vanishing of theta constants is known well, and the worst cases are the reducible and the hyperelliptic, which can be treated by hand, we have

Corollary 16. For $g=3,4$ the map $\mathbb{P}$ grTh is injective on $\mathcal{A}_{g}^{4,8}$.

We also prove the injectivity of $\mathbb{P} g r T h$ on generic Jacobians: notice that it does not directly follow from theorem 1 a priori as the Jacobians may not be the "generic" abelian varieties.

Proof of corollary 2. From the proof of theorem 1 we see that the map $\operatorname{PgrTh}$ is injective at $\tau$ if none of the theta constants at $\tau$ vanish. However, it is known classically from the works of Riemann that no theta constant vanishes identically on $\mathcal{J}_{g}$ Since $\mathcal{J}_{g}$ is irreducible, the subset of $\mathcal{J}_{g}$ where no theta constants vanish is Zariski open, and there the injectivity of $\mathbb{P} g r T h$ holds.

\section{INJECTIVITY ON THE TANGENT SPACE}

Now let us show that $\mathbb{P g r T h}$ is smooth. Let us study the situation in general terms first.

Lemma 17. Suppose $\mathbb{P} f: X \rightarrow G r(k, N)$ is an analytic map of a complex variety to the grassmannian, locally near some $p \in X$ given by $f: x_{1} \ldots x_{M} \rightarrow f_{1}(x), \ldots f_{k}(x)$, where $x_{i}$ are the local coordinates near $p$, and each $f_{i}$ is a vector in $\mathbb{C}^{N}$. Then $\left.\mathrm{dP} f\right|_{p}$ is injective if and only if for all $v \in \mathbb{C}^{M} \backslash\{0\}$ at least for one $I$ the vector $\partial_{v} f_{I}(0)$ is linearly independent with $\left(f_{1}(0), \ldots, f_{k}(0)\right)$. In particular it is injective if the vectors $\left(f_{1}(0), \ldots, f_{k}(0), \partial_{x_{1}} f_{I}(0), \ldots, \partial_{x_{M}} f_{I}(0)\right)$ are linearly independent for some $I$.

Proof. Indeed let us consider the linearization of $f$ near $p$; for $v \in$ $\mathbb{C}^{M}$ infinitesimally small we have $f(v)=\left(f_{1}(0)+\partial_{v} f_{1}(0), \ldots, f_{N}(0)+\right.$ $\left.\partial_{v} f_{N}(0)\right) .\left.\quad \mathrm{d} \mathbb{P} f\right|_{p}$ is non-degenerate iff it does not map any tangent 
vector $v$ to zero, i.e. if $f(v)$ represents a point in the grassmannian different from $f(0)$ for all $v$. This is equivalent to saying that for any $v$ at least one of the vectors making up $f(v)$ does not lie in $\mathbb{P} f(0)$, i.e. that at least one $\partial_{v} f_{I}(0)$ is linearly independent with $\left(f_{1}(0), \ldots, f_{k}(0)\right)$.

Now if $\left(f_{1}(0), \ldots, f_{k}(0), \partial_{x_{1}} f_{I}(0), \ldots, \partial_{x_{M}} f_{I}(0)\right)$ are linearly independent for some $I$, then it implies that any linear combination

$$
\partial_{v} f_{I}(0)=\sum v_{j} \partial_{x_{j}} f_{i}(0)
$$

is linearly independent with $\left(f_{1}(0), \ldots, f_{k}(0)\right)$ for all $v$.

Proof of theorem 3: injectivity of $\mathrm{dP}$ grTh on tangent spaces. We use the above lemma for $f=g r T h, k=g, M=g(g+1) / 2$ and $N=$ $2^{g-1}\left(2^{g}-1\right)$. In SM96] it is stated (Theorem 2b) that $\mathbb{P} g r T h$ is an immersion away from $\Gamma_{g}$-conjugates of points that are reducible as a product of a one-dimensional and a $g-1$-dimensional abelian variety. The proof there proceeds by showing that the rank of the matrix $\left(\operatorname{grTh}(\tau), \partial_{\tau_{i j}} g r T h(\tau)\right)$, (i.e. of $\left(f_{i}(0), \partial_{x_{j}} f_{i}(0)\right)_{\text {all } \mathrm{i}, \mathrm{j}}$ in the notations of the lemma 17] is maximal exactly for points that are not one-reducible. The maximality of this rank implies the maximality of the rank of any submatrix, in particular the one for which we need linear independence to apply lemma 17. However, the converse, implicitly assumed in SM96, is in fact not true: it would be requiring in addition to the lemma that $\left(\partial_{x_{j}} f_{i}(0)\right)$ are linearly independent, which is not necessary.

Thus the argument in SM96 shows that $\mathbb{P} g r T h$ is an immersion away from $\mathcal{H}_{1} \times \mathcal{H}_{g-1}$, and we only need to deal with $\tau=\left(\begin{array}{cc}\lambda & 0 \\ 0 & \tau^{\prime}\end{array}\right)$. In this case write $[\varepsilon, \delta]=\left[\varepsilon_{1} \varepsilon^{\prime}, \delta_{1} \delta^{\prime}\right]$, and let indices $i, j, I$ always be greater than one. Then

$\partial_{z_{1}} \theta\left[\begin{array}{l}\varepsilon \\ \delta\end{array}\right](\tau)=\partial_{z_{1}} \theta\left[\begin{array}{l}\varepsilon_{1} \\ \delta_{1}\end{array}\right](\lambda) \theta\left[\begin{array}{l}\varepsilon^{\prime} \\ \delta^{\prime}\end{array}\right]\left(\tau^{\prime}\right) ; \partial_{z_{I}} \theta\left[\begin{array}{l}\varepsilon \\ \delta\end{array}\right](\tau)=\theta\left[\begin{array}{l}\varepsilon_{1} \\ \delta_{1}\end{array}\right](\lambda) \partial_{z_{I}} \theta\left[\begin{array}{l}\varepsilon^{\prime} \\ \delta^{\prime}\end{array}\right]\left(\tau^{\prime}\right)$

Using the heat equation, for $\left.\mathrm{d} \partial_{z_{1}} \theta\left[\begin{array}{l}\varepsilon \\ \delta\end{array}\right]\right|_{\tau}$ we have

$$
\begin{gathered}
\partial_{\tau_{i j}} \partial_{z_{1}} \theta\left[\begin{array}{l}
\varepsilon \\
\delta
\end{array}\right]=\partial_{z_{1}} \theta\left[\begin{array}{l}
\varepsilon_{1} \\
\delta_{1}
\end{array}\right] \partial_{\tau_{i j}} \theta\left[\begin{array}{l}
\varepsilon^{\prime} \\
\delta^{\prime}
\end{array}\right] ; \partial_{\tau_{11}} \partial_{z_{1}} \theta\left[\begin{array}{l}
\varepsilon \\
\delta
\end{array}\right]=\partial_{z_{1}}^{3} \theta\left[\begin{array}{l}
\varepsilon_{1} \\
\delta_{1}
\end{array}\right] \theta\left[\begin{array}{l}
\varepsilon^{\prime} \\
\delta^{\prime}
\end{array}\right] \\
\partial_{\tau_{1 i}} \partial_{z_{1}} \theta\left[\begin{array}{l}
\varepsilon \\
\delta
\end{array}\right]=\partial_{\tau_{11}} \theta\left[\begin{array}{l}
\varepsilon_{1} \\
\delta_{1}
\end{array}\right] \partial_{z_{i}} \theta\left[\begin{array}{l}
\varepsilon^{\prime} \\
\delta^{\prime}
\end{array}\right] .
\end{gathered}
$$

What are the possible linear relations among these vectors? Arrange the vectors into a matrix and split this matrix into two corresponding to whether $\left[\varepsilon^{\prime}, \delta^{\prime}\right]$ is odd or even. We notice that all derivatives above are non-zero only for one parity of $\left[\varepsilon^{\prime}, \delta^{\prime}\right]$ (i.e. every column of the 
matrix has non-zero elements only in one of the two submatrices), and thus the matrix of vectors is in $2 \times 2$ submatrix block form, and we can compute the rank by adding the ranks of the blocks.

For $\left[\varepsilon^{\prime}, \delta^{\prime}\right]$ odd, the only non-zero elements of the corresponding row are $\partial_{z_{i}} \theta\left[\begin{array}{l}\varepsilon \\ \delta\end{array}\right]$ and $\partial_{\tau_{1 i}} \partial_{z_{1}} \theta\left[\begin{array}{l}\varepsilon \\ \delta\end{array}\right]$, which are independent because all $\partial_{z_{i}} \theta\left[\begin{array}{l}\varepsilon^{\prime} \\ \delta^{\prime}\end{array}\right]$ are non-collinear $\left(\operatorname{as} \operatorname{gr} T h\left(\tau^{\prime}\right)\right)$ and the matrix $\left(\theta\left[\begin{array}{l}\varepsilon_{1} \\ \delta_{1}\end{array}\right], \partial_{\tau_{11}} \theta\left[\begin{array}{l}\varepsilon_{1} \\ \delta_{1}\end{array}\right]\right)$ has maximal rank, two.

On the other hand, if $\left[\varepsilon^{\prime}, \delta^{\prime}\right]$ is even, then $\partial_{z_{1}} \theta\left[\begin{array}{l}\varepsilon \\ \delta\end{array}\right]$ and $\partial_{\tau_{i j}} \partial_{z_{1}} \theta\left[\begin{array}{l}\varepsilon \\ \delta\end{array}\right]$ are independent because the matrix $\left(\theta\left[\begin{array}{l}\varepsilon^{\prime} \\ \delta^{\prime}\end{array}\right], \partial_{\tau_{i j}} \theta\left[\begin{array}{l}\varepsilon^{\prime} \\ \delta^{\prime}\end{array}\right]\right)$ has maximal rank, while $\partial_{z_{1}}^{3} \theta\left[\begin{array}{l}\varepsilon \\ \delta\end{array}\right]$ is proportional to $\partial_{z_{1}} \theta\left[\begin{array}{l}\varepsilon \\ \delta\end{array}\right]$.

Thus using the above lemma we see that if $v \notin \mathbb{C} \partial_{\tau_{11}}$, the vectors $\left(f_{1}(0), f_{i}(0), \partial_{v} f_{1}(0)\right)$ are linearly independent. By the lemma, to prove the injectivity of $d \mathbb{P} f$ we then need to show that for $v=\partial_{\tau_{11}}$ the vectors $\left(f_{1}(0), f_{i}(0), \partial_{v} f_{I}(0)\right)$ are linearly independent. Indeed we compute

$$
\partial_{\tau_{11}} \partial_{z_{I}} \theta\left[\begin{array}{l}
\varepsilon \\
\delta
\end{array}\right](\tau)=\partial_{\tau_{11}} \theta\left[\begin{array}{l}
\varepsilon_{1} \\
\delta_{1}
\end{array}\right](\lambda) \partial_{z_{I}} \theta\left[\begin{array}{l}
\varepsilon^{\prime} \\
\delta^{\prime}
\end{array}\right]
$$

which is linearly independent with $\partial_{z_{I}} \theta\left[\begin{array}{l}\varepsilon \\ \delta\end{array}\right]$ because $\left(\theta\left[\begin{array}{l}\varepsilon_{1} \\ \delta_{1}\end{array}\right], \partial_{\tau_{11}} \theta\left[\begin{array}{l}\varepsilon_{1} \\ \delta_{1}\end{array}\right]\right)$ has maximal rank, and with $\partial_{z_{1}} \theta\left[\begin{array}{l}\varepsilon \\ \delta\end{array}\right]$ because one is zero for $\left[\varepsilon^{\prime}, \delta^{\prime}\right]$ odd, and the other — for $\left[\varepsilon^{\prime}, \delta^{\prime}\right]$ even.

\section{ACKNOWLEDGEMENTS}

We are very grateful to Lucia Caporaso and Edoardo Sernesi for bringing the subject to our attention and explaining to us their work on theta-hyperplanes, which inspired this article. We would also like to thank the organizers of the Complex Analysis meeting in Oberwolfach in August 2002 for bringing professor Caporaso and the first author to the same place at the same time, and thus making this work begin.

\section{REFERENCES}

[Ar872] S. Aronhold, Sur les vingt-huit tangentes doubles d'une courbe du quatrième degré. Nouv. Ann. (2) XI. 438-443. (1872). 
[Ca01] L. Caporaso, On modular properties of odd theta-characteristics, Advances in algebraic geometry motivated by physics, 101-114, Contemp. Math., 276, Amer. Math. Soc., Providence, RI, 2001

[CS00] L. Caporaso, E. Sernesi, Recovering plane curves from their bitangents, J. Algebraic Geom. 12 (2003), no. 2, 225-244.

[CS02] L. Caporaso, E. Sernesi, Characterizing curves by their odd thetacharacteristics, math.AG/0204164

[EL97] L. Ein, R. Lazarsfeld, Singularities of theta divisors and the birational geometry of irregular varieties, J. Amer. Math. Soc. 10 (1997), no. 1, 243258.

[Fa79] J. Fay, On the Riemann-Jacobi formula, Nachr. Akad. Wiss. Gottingen Math.-Phys. Kl. II 1979, no. 5, 61-73

[Fr885] F. Frobenius, Uber die constanten Factoren der Thetarheinen, J. Reine Angew. Math. 98 (1885) 244-265

[Ig72] J.-I. Igusa, Theta functions. Die Grundlehren der mathematischen Wissenschaften, Band 194. Springer-Verlag, New York-Heidelberg, 1972.

[Ig80] J.-I. Igusa, On Jacobi's derivative formula and its generalizations, Amer. J. Math. 102 (1980), no. 2, 409-446

[SM83] R. Salvati Manni, On the nonidentically zero Nullwerte of Jacobians of theta functions with odd characteristics, Adv. in Math. 47 (1983), no. 1, 88-104.

[SM94] R. Salvati Manni, Modular varieties with level 2 theta structure, Amer. J. Math. 116 (1994), no. 6, 1489-1511.

[SM96] R. Salvati Manni, On the differential of applications defined on the moduli space of p.p.a.v. with level theta structure, Math. Z. 221, 231-241 (1996)

[Sa83] R. Sasaki, Modular forms vanishing at the reducible points of the Siegel upper-half space, J. Reine Angew. Math. 345 (1983), 111-121

E-mail address: sam@math.princeton.edu and salvati@mat.uniroma1.it

Mathematics Department, Princeton University, Fine Hall, Washington Road, Princeton, NJ 08544, USA and Dipartimento di Matematica, Università di Roma, Piazzale Aldo Moro, 2, I-00185 Roma, Italy 